\title{
Educación, humanidades y opción decolonial
}

\author{
Andrés Argüello Parra*
}

\begin{abstract}
Resumen
El artículo se basa en la opción decolonial como una propuesta de integración de perspectivas educativas y sociales adecuada para apreciar los universos de comprensión alternativos a la Modernidad desde un "paradigma-otro" que permita recoger mejor las nuevas preguntas y las nuevas problematizaciones en torno al ser humano y su relación con el cosmos. Para ilustrar esto, el texto se estructura en dos grandes secciones: en primer lugar, expone las condiciones que permiten identificar un marco comprensivo de las humanidades en el tercer milenio. En la segunda sección, se plantean los elementos de una pedagogía decolonial que contribuya a promover desde la educación una racionalidad alternativa de vida y humanidad.

Palabras-clave: Pedagogía, Decolonialidad, Humanidades.
\end{abstract}

\section{Educação, humanidades e opção decolonial}

\section{Resumo}

$\mathrm{O}$ artigo baseia-se na opção decolonial como uma proposta de integração de perspectivas educativas e sociais útil para apreciar os universos de entendimento alternativos à Modernidade desde um "paradigma-outro" que permita recolher melhor as novas perguntas e as novas questões em torno do ser humano e a sua relação com o cosmos. Para mostrar isto, o texto se estrutura em duas partes: a primeira, expõe as condições que permitem identificar um marco compreensivo das humanidades no terceiro milénio. Na segunda parte, propõem-se os elementos de uma pedagogia decolonial que contribua a promover uma racionalidade alternativa de vida e humanidade a partir da educação mesma.

Palavras-chave: Pedagogia; Decolonialidade; Humanidades

\section{Education, humanities and decolonial option}

\section{Abstract}

The article is founded on decolonial option's as a proposal to integrate different educational and social perspectives, which is relevant to value alternative universes of Modern comprehension from a "paradigmother" [paradigma-otro] as to better reflect on new questions and new problematizations with regards to human being and its relations with the cosmos. This article is structured around two major aspects: first, it sets out the conditions that allow the identification of a comprehensive framework of humanities for the third millennium and, second, it proposes elements based on decolonial pedagogy that would contribute to promote, from education, an alternative rationality on life and humanity.

Key-words: Pedagogy; Decolonial thinking; Humanities

\section{Introducción}

El avance de la segunda década del siglo

XXI ha evidenciado progresivamente la transformación de los distintos órdenes humanos y la manera de comprenderlos desde otras racionalidades allende los modelos explicativos lineales dominantes en la modernidad occidental. En el presente artículo se discute el desafío de la resignificación de las humanidades en el ámbito de la misión de la Universidad no sólo como espacio matriz del desarrollo científico y tecnológico sino como servicio al florecimiento de la cultura, la democracia crítica y la coexistencia de los seres.
Ello exige situarse en una racionalidad que no privilegie sólo el ámbito instrumental o productivo de lo humano sino ante todo la dimensión relacional e integradora de lo múltiple, de lo complejo, tal como puede hallarse en los aportes de las perspectivas decoloniales.

El texto se desarrolla a partir de dos grandes componentes. En primer lugar, se ilustra el marco comprensivo de las humanidades en la "encrucijada" de la revolución tecnológica, lo cual implica una visión que supere el tratamiento esencialista clásico fundado en los principios de universalidad y teorización. En el segundo momento, se plantean algunos apuntes para 
comprender la educación desde una opción decolonial, sus desafíos e implicaciones en el marco de la construcción de una cultura de la paz.

\section{Marco comprensivo de las humanidades}

Es preciso constatar, como punto de partida, que la llamada crisis de las humanidades hoy es, ante todo, una crisis de lo humano. No sólo de sus aspiraciones éticas más representativas sino de su misma racionalidad, de la manera de entender el fenómeno de la existencia humana y de sus complejas relaciones con la vida planetaria.

Las tensas relaciones del humanismo principalmente el de herencia greco-latina y judeocristiana - con el mundo globalizado, a causa de la revolución tecnológica y la geopolítica del conocimiento, expresan una inmanentización de las aspiraciones últimas de lo humano donde los "hombres usarán la tecnología para transformarse en dioses", como lo ha indicado el historiador israelí Noah Harari, a propósito de su reciente publicación "Sapiens: De animales a dioses, breve historia de la humanidad" (TIBBLE, 2016).

Se trata, entonces, de una crisis de sentido fundada en el desplazamiento paradigmático del modo de conocer "humanista": El modelo esencialista del hombre bueno, erudito $\mathrm{y}$ enciclopédico (homo sapiens) se confronta con la aspiración cibernética del homo digitalis.

Es este control de la existencia por el diseño inteligente capaz de alterar el orden humano de manera irreversible, el punto de partida de los llamados post y transhumanismos, donde - en opinión de este autor - "el hombre decide que ya es hora de dejar de ser hombre. Se desprende de sus cadenas biológicas y contempla, en los albores del siglo XXI, desgajar las leyes de la selección natural y sustituirlas por las del diseño inteligente. Juega a ser Dios, a producir ciborgs, a revivir animales extintos, a descargar su conciencia en un computador. Se desdibujan los límites. El homo sapiens se deshumaniza y se encamina, emocionado, a culminar su proyecto de vencer la muerte" (ibídem, p. 15).

En un marco similar de comprensión se encuentran los trabajos del científico social sur coreano Byung-Chul Han (2012) y sus valiosas contribuciones para analizar los nuevos dispositivos del poder impulsados por el capitalismo neoliberal, los cuales dan acceso a la dimensión psíquica, para transformarla en una singular fuerza de producción. Una vez conquistada la psique humana sea por el miedo, la irracionalidad - entendida como avance de la insignificancia - o la expansión del deseo, toda la inteligencia y la voluntad terminan al servicio de esa fuerza totalizante de control. La interioridad colonizada es la interioridad usufructuada. Esta es justamente la tesis más iluminadora en el ensayo La sociedad del cansancio: Se pasa progresivamente de la alienación y explotación hecha por otros, como fue denunciado en la crisis del trabajo durante la revolución industrial, a una autoexplotación voluntaria consciente o inconsciente.

Es la dimensión de la Psicopolítica donde "la decadencia general de los valores erosiona la cultura del respeto. Los modelos actuales carecen de valores interiores. Se distinguen sobre todo por cualidades externas" (HAN, 2014, p. 18). Este auge de la exteriorización, del espectáculo y del voyeurismo mediático, se alimenta y se robustece, principalmente por los contenidos derivados del modelo económico, tecnocrático e ideológico dominante, que a pesar de sus desestabilizaciones lucha por su prolongación y permanencia.

En la Psicopolítica, la revolución tecnológica, así como la producción cibernética de lo humano, fundada en los intereses del capital, deriva hacia la "voluntaria esclavitud de soledades" donde la crisis del enfoque clásico de las humanidades se manifiesta en dos bloques característicos: el Imperio del consumo y la hegemonía del pensamiento único.

a) Imperio del consumo

En el avance del tercer milenio, se han sofisticado los mecanismos para la producción del sujeto neoliberal. Primero, a partir de un cuerpo de doctrina o "dogmática neoliberal - La "nueva razón del mundo" Laval y Dardot - y segundo, por un conjunto de prácticas sociales que hace explícita la globalización del capital y los procesos de re-occidentalización fundados en el dinero.

Así, la empresa es promovida a la categoría de modelo de subjetivación; es una manera de construir sociedad y de fundar instituciones cuya prolongación se asegura desde la escuela, desde los modelos educativos que explícita o tácitamente reflejan la cultura y el espíritu del mercado. Tal vez la expresión más visible de este imperio del consumo, motor de la "cultura del descarte" alertada de modo singular por el Papa Francisco (2015, n. 43), sea el establecimiento de un sistema de relaciones que cosifican la noción de otredad, vaciando al otro de su misterio y de su posibilidad: 
"El capitalismo avanzado es esencialmente destructor de la dimensión colectiva de la existencia" (DÍEZ, 2014, p. 44).

El consumo se erige, pues, como hiperbolización del individuo que anula o por lo menos disuade el carácter simbólico-relacional de la existencia. Al reducir el encuentro a depredación y aprovechamiento, monetariza el papel del hombre en la naturaleza, vacía la condición mítica y dispersa la fuerza integradora de la significación cósmica, propia de una antropología basada en la complejidad, la reciprocidad y la complementariedad de los seres. Se establece, así, una univocidad de pensamiento y de conducta según se expone a continuación.

b) Hegemonía del pensamiento único

El consumo es negación de lo múltiple desde la prevalencia de la racionalidad económica. Existe una reactualización del pensamiento único expresado en un nuevo alcance del dualismo nosotros-ellos. Se entiende aquí por Pensamiento único el horizonte de comprensión heredado de la modernidad, cuya pretensión de universalidad se traduce en normalización de arquetipos diseñados localmente, que envuelven la dimensión social, cultural, económica, intelectual, pedagógica, política, en fin, la totalidad del ecosistema humano.

El pensamiento único se expresa, básicamente, como negación de la diversidad humana y cósmica en lo social que es uno de los sustratos más vigorosos de la razón de ser de las humanidades contemporáneas. Bien lo ha recordado la Declaración de la UNESCO sobre la Diversidad Cultural (art. 1) al definirla como "[...] patrimonio común de la humanidad. [...] tan necesaria para el género humano como la diversidad biológica para los organismos vivos".

En este punto se pueden destacar, al menos seis campos representativos de esta hegemonía de cosmovisión, que son fundamentales para la refundación de las humanidades en la encrucijada de las transformaciones históricas contemporáneas:

- 1. Sujeto blanco. Expresa el dominio del pensamiento único de la raza blanca noratlántica frente a las múltiples constituciones étnicas, que derivan en criollismo dependiente, prejuicio racial (indígena, afrodescendiente) y veneración irreflexiva de lo extranjero como cualitativamente superior que lo propio (xenofilia radical y etnocentrismo práctico).
- 2. Sujeto varón-androcéntrico. Es el relato del pensamiento único frente a la condición de género, que se expresa particularmente en el patriarcalismo y su consecuente subvaloración axiológica, minusvalía ciudadana y postergamiento social de la mujer.

- 3. Sujeto heterosexual. Constituye el dominio del pensamiento único frente a la orientación sexual que sólo legitima la relación con el sexo opuesto sobre la negación del florecimiento de las múltiples sexualidades como mundos posibles de identidad y de organización social.

- 4. Sujeto cristiano y católico. Es la manifestación del pensamiento único en el plano de las creencias [religiosas] validadas como comúnmente aceptadas desde un modelo de cristiandad donde la misión o la conversión del otro anula la multiplicidad de los modos de creer y de no creer tan característicos en las sociedades contemporáneas.

- 5. Sujeto aristócrata y capitalista. Manifiesta la prevalencia del pensamiento único frente a los modos de producción económica (radicalismo capitalista neoliberal) y el sostenimiento de la organización social propia del statu quo, que incluye expresiones variadas de la manipulación del derecho, de los valores supremos del Estado y el acatamiento de las instituciones.

- 6. Sujeto amo de la naturaleza. Es el relato del dominador que traduce al pensamiento único en la relación depredadora de lo humano frente a los animales, los bienes naturales, las leyes del cosmos y la biodiversidad.

La conjunción del imperio del consumo y de la hegemonía del pensamiento único produce la distopía del poshumanismo, es decir, el marco de una sociedad considerada indeseable en el ciclo póstumo de las humanidades, y que podría sintetizarse en las siguientes tipificaciones:

- Es un tiempo de incertezas, caracterizado por la soledad interior, el hastío existencial, el vacío frente al mundo moderno cuyos axiomas son insuficientes para responder a las necesidades $y$ tribulaciones del siglo XXI.

- Desconfianza integral por personas, 
ideologías e instituciones, que expresa los grandes miedos de la sociedad planetaria, los cuales van al ritmo de los conflictos globales, producto de la acción humana: nuevos armamentismos, amenazas nucleares, pandemias, fractura de las seguridades ciudadanas, desertificación del planeta. El resultado es una crisis de confianza como valor fundador de la estabilidad y armonía de las sociedades.

- Rebrote de fanatismos de todo tipo, principalmente los de inspiración política y religiosa que, en su versión más radical, se convierten en amenaza cierta de exterminio del otro. Conlleva, entonces, a la negación de la pluralidad, la radicalización de las xenofobias donde la alteridad es amenaza y objeto de consumo.

- Sobre-exposición de la vida privada, cierto exhibicionismo mediático impulsado por los artificios del homo digitalis, que conlleva a identidades diluidas y desapropiadas. No hay posesión de sí mismo. Como sugiere Orwell, en su obra 1984, podemos perder nuestra libertad en cualquier momento.

- En suma, el antropoceno global se caracteriza por una crisis humanitaria generalizada, donde los dramas más acuciantes de la historia actual no sólo se tornan recurrentes sino que se profundizan y se sostienen sin una atención reparadora integral. La relación de las humanidades con la historia deviene quebradiza y mudable en sus propios fundamentos.

\section{Humanidades y educación desde una perspectiva decolonial}

El panorama descrito exige refundar crítica y contextualmente la razón de ser de las humanidades y su papel en la educación, sin añoranzas anacrónicas ni pietismos estériles. Sin añoranzas anacrónicas porque no basta sugerir el retorno a las formas clásicas occidentales de la comprensión humana, tan insuficientes hoy como su misma ausencia. Sin pietismos estériles, porque es preciso consolidar el estatuto secular de la reflexión humanística desde el conjunto de valores cívicos que coadyuven a la construcción de sociedades democráticas. De esta forma, el punto de partida para abordar la interacción humanidades-educación es la insuficiencia del modelo de la racionalidad escolástica y moderna ante la complejidad de los problemas del mundo contemporáneo.

En este punto, tal como ha sido analizado por estudiosos del tema (DE SOUSA, 2002; LEFF, 2000; ESCOBAR, 2012), "la habilidad de la modernidad para proveer soluciones a los problemas modernos ha sido crecientemente comprometida. En efecto, puede ser argumentado que no existen soluciones modernas a muchos de los problemas de hoy" (ESCOBAR, 2012, p. 49).

Es preciso, entonces, co-construir una racionalidad-otra - y no sólo "otra racionalidad"para comprender los sentidos y significados de un mundo que no se puede entender sólo desde la metafísica occidental o la teología dogmática o el simple retorno a las humanidades clásicas (grecolatinas, renacentistas e ilustradas).

De esta manera, si la crisis de las humanidades es la crisis misma de lo humano, se propone la plataforma epistémica de la decolonialidad como una cosmovisión establecida en el reverso de la modernidad. Es una opción, entre muchas otras, (desarrollada por el grupo modernidad-colonialidad) que no pretende ser definitiva ni única pero que se integra a los esfuerzos para favorecer la comprensión de las realidades históricas, humanas y planetarias multirreferenciales, que caracterizan la historia presente.

A partir de esta base comprehensiva y dentro de los sistemas emergentes de pensamiento que afectan hoy todos los ámbitos de la existencia, se propone el enfoque de la educación humanistacompleja, y no sólo humanista clásica (en un sentido greco-latino y renacentista europeo), o humanista ciudadana (en un sentido liberal ilustrado).

Tomando en cuenta las reflexiones del grupo modernidad-colonialidad, complementadas con el aporte de Edgar Morin (2000) en la reunión de intelectuales de la UNESCO basada en la pregunta ¿Existirá el siglo XXI?, una educación humanista tejida en lo plural, abarcaría al menos cuatro movimientos complementarios (ARGÜELLO, 2015), a saber:

a) Decolonizar el saber/ reforma del pensamiento:

El eje fundante de todo campo de acción social es la reforma del pensamiento, esto es, afectar la forma de conocer, de valorar $y$ 
representar, antecedido por la forma de enseñar. Para tal fin es prioritario superar las epistemologías dicotómicas fundadas en la división sujeto-objeto y las didácticas lineales: "El gran reto para el conocimiento, la educación y el pensamiento de nuestro siglo - que adquirirán cada vez mayor importancia en el próximo - es la contradicción entre los problemas cada vez más globales, interdependientes y planetarios, por una parte, y nuestra forma de conocer, que es cada vez más fragmentada, parcelada y compartimentada, por otra parte" (MORIN, 2000, p. 966).

En consecuencia es necesario transitar hacia un tipo de pensamiento que evite las cegueras del conocer producto de la superstición, el dogmatismo, la irreflexión o la ingenuidad ideológica con base en las epistemologías basadasen-lugar (ESCOBAR, 2012). Optar por conocimientos situados es viabilizar aquellos saberes encarnados en las conexiones del contexto humano y social, con las construcciones identitarias en situación y los relatos locales.

Para ello es menester revisar los criterios de validez del conocimiento y posicionar la interculturalidad decolonial y crítica. La interculturalidad decolonial es el reconocimiento del otro en su ser, implica confrontar los discursos falaces de integración promovidos por los modelos hegemónicos, o las voces de aceptación tolerante pero sin implicación práctica en el ordenamiento de la historia colonial. La simple aceptación o "tolerancia" del otro no expresa la reciprocidad intercultural; tampoco la "inclusión" mesiánica o tecnocrática que integra para diluir.

Es el reconocimiento de que no hay una forma canónica de ser, una identidad universal, sino una trama de multiplicidades en relación. Por eso el cultivo de la diversidad "es un imperativo ético, inseparable del respeto de la dignidad de la persona humana" (UNESCO, Declaración sobre la Diversidad Cultural, a. 4). Reconocer la dignidad del ser humano es reconocer los muchos modos de serlo. Que el otro pueda ser quien es y en cuanto tal ser un productor de conocimientos, legitimado en la relación sujeto-sujeto desde una perspectiva dialógica.

En ese horizonte se hará vital apostar por menos Europeísmo y más indigenismo y ancestralidad; menos Kant y más Nezahualcóyotl:

\footnotetext{
"Por fin lo comprende mi corazón:

Escucho un canto,

Contemplo una flor:
}

\section{¡Ojalá no se marchiten!’.}

\section{b) Decolonizar el ser /enseñar la condición humana}

Una vez el relato dominante de la modernidad (a través de sus dispositivos de saberpoder-organización) produce la modelación intencionada de los comportamientos y diseños existenciales, la enseñanza de la condición humana surge aquí como un criterio fundamental de conciencia emancipatoria y auto-afirmación.

Puesto que a la modernidad colonial no basta colonizar cuerpos, pensamientos y conductas, sino que emprende, a su vez, la colonización de las subjetividades mediante la normalización del ser, decolonizar la propia naturaleza del sujeto se entiende como la construcción de un proyecto de afirmación de la identidad en clave ética que sostenga las múltiples relaciones hombre-cosmos. Es la opción decolonial orientada a "reconocer que el conjunto de la humanidad vive en una comunidad de destino sometida al mismo problema de vida y de muerte" (MORIN, 2000, p. 966).

En las prácticas educativas, enseñar la condición humana exige revisar la manera como se comprende y se procesa la realidad: modificar concepciones debe implicar la modificación efectiva de comportamientos ante la vida, por ende, asumir compromisos situados. Decolonizar el ser es construir una ética contextual que parta de la comprensión de las tradiciones concretas, de una razón práctica de lo particular, desde los individuos y las comunidades concretas, para la generación de un proyecto planetario de humanidad.

Para tal fin se requiere producir escenarios que alberguen la negociación de significados característico del pluralismo humanista-cósmico. Empero, esta negociación de significados sólo ocurre en el encuentro vital, por eso la decolonización del ser sintoniza metodológicamente con las apuestas de la perspectiva biográfica en la investigación social.

Las pedagogías decoloniales trabajan desde y con el aporte de las historias locales. Es la única posibilidad de (re) conocer las zonas de silencio impuestas por las hegemonías del conocimiento y del poder. Por ello, el potencial de la biografía es determinante al construir las genealogías o locus enuntiationis en situaciones coloniales pues revela el proceso de los lugares de dominación no sólo desde la historia personal del sujeto sino también desde los roles sociales $y$ las funciones 
institucionales en las coordenadas de poder, género, raza, clase social, sistemas de creencias, afiliación política y otros vectores de subjetivación.

Constituir cierta manera de ser allende las univocidades de la subjetivación occidental sólo es posible por la incursión epistémica y práctica en los territorios simbólicos desconocidos de la radical alteridad, es decir, en la capacidad de conocer e interpretar cómo se han tejido los universos del olvido, la segregación y las relaciones centro-periferia como prácticas normalizadas de las sociedades modernas.

c) Decolonizar las prácticas socio-culturales y la relación con el cosmos/Enseñar a vivir

Desde el enfoque humanista-complejo de la educación es preciso tener en cuenta que "el humanismo no tiene como ambición el dominio. Tiene como misión la convivencia en la Tierra" (MORIN, 2000, p. 967). La opción decolonial de las humanidades en la educación concuerda con este postulado al considerar que "No se trata solamente de aprender técnicas, conocimientos y sistemas productivos, sino de mantener relaciones con otros y consigo mismo" (Ibídem, p. 967).

Este es el presupuesto para un proyecto social de educación tendiente a conocer la complejidad de los seres humanos y de sus relaciones, a formar ciudadanos de la Tierra y no sólo mano de obra acrítica para los intereses del mercado. La tarea es explorar los "mundos-otros" desde la atribución de un singular poder social a los actores de las comunidades educativas.

La educación humanista que trabaja por la decolonización de las prácticas socio-culturales de la modernidad, hace suya la máxima zapatista decidiéndose a construir un mundo donde quepan muchos mundos. En este programa pedagógico del enseñar a vivir, la esencia humana proviene principalmente de la misma existencia confiriendo un papel central al registro inductivo de la experiencia vital, de la experiencia histórica e institucional.

Uno de los campos más representativos, que cumple un papel vertebrador de las prácticas coloniales en la sociedad, es el de la ley y el derecho. En este plano, la educación para la paz y los derechos humanos como práctica social y política se entiende fundamentalmente como experiencia humana efectiva, campo que hace visible el debate de algunos hermeneutas latinoamericanos del derecho quienes señalan la tensión entre derechos humanos como abstracción idealista y derechos humanos como situación histórica. El profesor Jesús Antonio de la Torre explica, a este propósito: "El gran problema que afronta el iusnaturalismo es el de su a historización, es decir, en reducirse a conceptos bonitos pero vacíos de contenidos reales. Esto es, se vuelven términos que encubren la injusticia, cuando en la realidad histórica concreta no se descubre al otro" (MARTÍNEZ, 2008, p. 44).

De este modo, la decolonizar las prácticas socio-culturales desde la acción educativa, conlleva el proceso de "hacer" históricos los conceptos ("iusnaturalismo histórico") como tarea social desde un enfoque de praxis e historización. $\mathrm{Si}$ bien deben existir los dispositivos institucionales del Estado que la favorezcan y la permitan, la construcción de la paz, como protoderecho de la política, comienza desde la base, desde las narrativas locales, desde las convicciones individuales traducidas en acción comunitaria.

En este horizonte, es necesario desarrollar una dimensión de las profesiones profundamente vinculante de la experiencia humana para hacer florecer, desde ahí, desde esa clave de humanidad, el ejercicio continuo del pensamiento crítico para no resultar cautivos de odios generacionales ni de ideologías sacralizadas. Todorov: "Hay que aspirar a una historia que escape al maniqueísmo e intente arrojar una mirada crítica y lúcida sobre el pasado [y el presente] de nuestra comunidad" (GASCÓN, 2015). El pensamiento crítico exige alejarse del dogmatismo y del nihilismo, de tener todas las respuestas hechas, o de no ser capaces de ofrecer ninguna, ambos favorecedores de la polarización que se cierra sobre sí misma.

d) Decolonizar las instituciones escolares y sus dispositivos pedagógicos: currículo, evaluación, organización / reforma de la escuela ante los retos de la paz

Para realizar una educación humanista en clave decolonial no basta estar en sintonía con el sistema social dominante o cumplir el mandato del Estado, que se adjudica las funciones de suprema inspección y vigilancia. En otras palabras, es insuficiente cumplir las funciones de conservación cultural si no hay una posición crítica y transformadora ante lo ya dado.

Optar por una educación humanista no es sólo declararla. Exige, fundamentalmente, superar la brecha entre el decir y el hacer, entre la declaración y la implementación, pues en no pocas oportunidades, las instituciones pueden generar discursos emancipatorios, por un lado, pero 
prácticas opresivas, por otro. Aquí debe producirse una clave histórico-pedagógica propia para afrontar el macro-relato escolar del rendimiento lineal, la competencia megalómana, la calidad capitalista y la eficacia industrial para determinar qué tipo de codificación institucional se está realmente asumiendo en las prácticas educativas concretas.

Esta apuesta decolonizadora de la escuela abarca la promoción de diseños curriculares de tipo trans-disciplinar y problémico, en horizonte bioético, como una manera de confrontación de las disciplinas fundadas tradicionalmente en las epistemologías del poder, al margen del conocimiento situado en las biografías particulares y las geografías locales (antropologías territoriales).

Para realizar este empeño se hace necesario favorecer un sistema curricular que desestimule la dominación del pensamiento único e impulse una didáctica decolonial que contemple propuestas concretas para la instauración de una perspectiva integradora en educación, esto es, lugares sociales para formar una solidaridad planetaria apoyada en la coexistencia, la reciprocidad y la dignidad de todos los seres desde la construcción de interrelaciones no-dominantes en la diferencia (DE LISSOVOY, 2010).

Cuando se habla de reforma en la escuela y especialmente de reforma humanista, hay que incentivar el papel contrahegemónico de los liderazgos, de quienes desempeñan funciones de autoridad en el sistema académico, el primero de ellos, el sujeto-maestro. Sencillamente porque la educación cambia efectivamente donde hay un/a maestro/a con voluntad de cambio, un/a directivo/a que deja hacer, un estudiante que confronta con creatividad y crítica.

Contra las nuevas manifestaciones del pensamiento único en la falacia del mundo global, es preciso declarar que la educación debe ser comprendida y accionada como tarea social y proyecto ético, y no sólo como un programa o una estrategia de adiestramiento y de acomodación a cierta forma hegemónica de poder, de poder o de directividad.

Ello implica una educación como comprensión y vivencia (es decir, como pensamiento crítico y voluntad creadora) de un sistema de valores deseables en la socialización de la vida cotidiana, del trabajo, de las asociaciones, de "la semiótica de la calle". La educación no es sólo la acción institucional de la escuela, no es sólo mandato social, implica contribuciones multifactoriales para reparar sinérgicamente los problemas sociales que afectan las historias reales de muchos sectores. Esto exige entender la educación como interlocutora política y no sólo como servidora lacaya de una nación o de una cultura occidental.

Este horizonte implica posicionar la educación en las coordenadas de la concordia inter-humana: las pedagogías para la paz se entienden como gestión razonada de los conflictos y no como ausencia de ellos. Decolonizar las instituciones y las prácticas educativas es aprender a vivir y a gestionar los conflictos y cómo éstos se podrían transformar en otra manera de relacionarse.

Supone abordar también el estudio transdisciplinario de las causas de la violencia multidimensional y no sólo de sus efectos; devaluar la violencia simbólica en la cotidianidad escolar (micro-violencias normalizadas) y socavar el vínculo violencia-economía desde una educación que desplace la "cultura del descarte" pues "el mercado liberal desregularizado y desarticulado es caldo de cultivo para egoísmos, odios y guerras. Ahí prosperan las fuerzas de separación, dispersión y muerte" (MORIN, 2014, p. 8).

La reconciliación no se entiende como la afinidad ingenua de voluntades sino como la supresión de los espirales de violencia que actúan como combustible de la venganza y la convierten en práctica infinita de muerte. Imposible no mencionar aquí el papel de la memoria y de la conciencia histórica pues conocer qué pasó es el primer eslabón de la garantía de no repetición. La memoria debe contribuir al deber de conocer la verdad y de hacer justicia. No es un dispositivo para la venganza (GASCÓN, 2015).

Las pedagogías generadoras de culturas de paz suponen comunidades educativas que ordenan el pensar, el ser y el actuar desde la solidaridad colectiva para construir civilidad y desarrollo integral en los territorios, en las periferias de las ciudades, en el compromiso cívico particular frente a las alteridades negadas. Es en tal sentido que Philippe Meirieu, afirma que debemos apostar por "un acto pedagógico que aporta una increíble esperanza social y política: la esperanza de una sociedad en la cual las relaciones entre los seres no se basarían en la violencia del mercado y en la eliminación del "rival más débil", sino en la búsqueda colectiva, a partir del respeto mutuo, de 
la verdad" (2011, p. 102).

\section{A manera de conclusión}

La reinvención educativa desde un sentido decolonial de las humanidades exige, en primer lugar, someter a examen los esquemas de significado y universos simbólicos desde los cuales se valora el mundo para diseñar un marco alternativo de interpretaciones que permitan comprender "el mundo que pasa" como el "mundo que nos pasa". Ello supone considerar la capacidad transformadora de la experiencia para afectar no sólo la pertinencia social de la educación sino el alcance mismo de sus finalidades. El arquetipo de la historia y el examen de la cotidianidad fraternizan en una epistemología dialogante con las biografías y los proyectos de memoria compartida que nos rodean: "El universo simbólico no puede existir sin los mundos de la vida y las relaciones cara-a-cara y sólo podemos conocerlo desde [sus] manifestaciones históricas" (MELICH, 1998, p. 44).

Plantear la importancia de las humanidades en la educación actual implica, entonces, asumir la multiplicidad de la condición humana; la certeza fiable de que no hay una acepción unívoca de ser hombre. Al mismo tiempo ello supone asumir las tensiones de una época que se ha reinventado en sus propósitos, aspiraciones y cotidianidades para apreciar las dimensiones de humanidad que no son accesibles a los registros cuantitativos de los datos: el ámbito de la construcción simbólica.

La opción decolonial exige quitar la última palabra a la hegemonía del mercado y sus violencias, junto con sus tradiciones de negación y ocultamiento. Al sostener que "la construcción significativa del mundo social es una construcción simbólica" (Ibidem, p. 44), busca dar lugar a un relato nuevo de sociedad donde sea reconocida "la razón de los vencidos".

La educación decolonial en cualquiera de sus acepciones, como fuerza socio-cultural capaz de afectar la (re)construcción de las civilizaciones, y de las historias, y de las tradiciones comunitarias, y las relaciones con el cosmos, ofrece la potencialidad de encaminar, en lo cotidiano, un proyecto de civilización bio-centrada.

No podría ser de otro modo en medio de una sociedad donde los monstruos de la barbarie, quizás duermen por momentos, pero nunca se han ido; donde cunden los necios, que decía Wilde, aquellos "que saben el precio de todo y el valor de nada"; donde dominan cotidianidades marcadas por las formas sociales de la mafia y el crimen; sociedades del atajo y del "yo no me dejo", donde el insulto y la descalificación reemplazan la discusión [...] para fomentar finalmente el estigma; donde se mata por cualquier nimiedad, se desfiguran personas con ácido, se saca provecho del más débil, seres humanos u otros animales; se extermina al adversario ideológico, se acomoda la ley y el derecho para pasar por encima de todos en razón de intereses exclusivamente privados. Cuadro eterno del horror en un contexto lacerado y depravado, sumido al imperio de los psicópatas de la muerte, con la complicidad de impunidades estructurales de una amañada e ineficaz administración de justicia, y de una creciente comercialización de los derechos fundamentales, que significa en últimas convertir la persona en mercadería.

Es momento, entonces, de favorecer una civilización donde se valore la conciencia íntima del sujeto como un santuario de la dignidad donde reside el criterio de una absoluta igualdad de reconocimiento y respeto. Momento para el elogio de la diferencia deudora de la convivencia, de la diversidad instalada en los intereses comunes necesarios en cada situación y escenario social. Lugar para confrontar la crisis de conciencia; la sofisticada estupidez que todo lo juzga desde la entelequia de la norma general.

Esta educación se parece más a una obra de arte que fusiona simultáneamente la irrealidad y el dato fáctico, la fantasmagoría y la información veraz. No es un simple manual de procedimientos donde todo se hace de manera programada y predecible. Es más un plan de ruta con ciertas coordenadas preestablecidas pero con opciones de cambio, de re-direccionamiento y de respuesta según cada caso. De ahí la importancia de no hacer del medio o del recurso una finalidad en sí misma. Los problemas centrales de la educación no son los del formato o los de la calidad organizacional o los de la innovación tecnológica, impuestos por un modelo económico dominante e idolatrado.

$\mathrm{Si}$ destacamos un conjunto de valores centrados en la capacidad autoreguladora de las personas, se daría lugar al desarrollo de imperativos éticos personales, cívicos y planetarios, con criterio autónomo y razonable. Sólo el sujeto capaz de sí puede ser dueño de su historia. Por eso, al impulsar las elecciones que privilegian la vida, no en abstracto, sino en cada persona, en cada ser viviente, suceso y situación, 
en el sentido común que comienza en la acción cotidiana y callejera, se favorece, sí, una autonomización, pero corresponsable en alteridades, temporalidades y vinculaciones. Desde esta perspectiva, la educación decolonial emerge como un correlato fáctico de la supervivencia que espera nuestra propia dedicación y compromiso.

\section{Referencias}

ARGÜELLO, A. Pedagogía decolonial: trazos para la construcción de un paradigma-otro desde la educación. En: Correo del Maestro, Revista para profesores de Educación Básica, v. 19, n. 226, p. 28-37, marzo 2015.

DE LISSOVOY, N. Decolonial pedagogy and the Ethics of the Global. En: Discourse: Studies in the Cultural Politics of Education, v. 31, n. 3, p. 279-293, 2010.

DÍEZ, E. La construcción educativa del nuevo sujeto neoliberal. En: El viejo topo, n. 320, p. 3947, sept. 2014.

ESCOBAR, A. Una minga para el post desarrollo: Lugar, medio ambiente $\mathrm{y}$ movimientos sociales en las transformaciones globales. Bogotá: Ediciones Desde Abajo, 2012.

FRANCISCO, Papa. Laudato Si. Sobre el cuidado de la casa común, 2015. Disponible en: file://C:/Users/CW13B/Downloads/249-810-1PB.pdf $>$. Acesso en: $20 \mathrm{dez} / 2016$.

GASCÓN, D. "La memoria tiene una potencia que la historia nunca alcanza". Entrevista con Tzvetan Todorov. En Letras Libres, n. 198, junio $2015 . \quad<$ Disponible en http://www.letraslibres.com/revista/dossier/lamemoria-tiene-una-potencia-que-la-historianunca-alcanza>. Acesso en: 20 dez/2016.
HAN, Byung-Chul. En el enjambre. Barcelona: Herder, 2014.

HAN, Byung-Chul. La sociedad del cansancio. Barcelona: Herder, 2012.

LEFF, E. Saber ambiental. México: Siglo XXI, 2000.

MARTÍNEZ, A. Derechos humanos, liberación y filosofía de la realidad histórica. En Teoria crítica dos direitos humanos no século XXI. Porto Alegre: EdiPUCRS, p. 15-45, 2008.

MEIRIEU, P. Carta a un joven profesor. ¿Por qué enseñar hoy? Barcelona, Grao, 2011.

MÉLICH, J-C. Antropología simbólica y acción educativa. Barcelona: Paidós, 1998.

MORIN, E. La finalidad del proceso educativo o la religación ética del sistema En: Acuerdo por lo superior 2034, Consejo Nacional de Educación superior, Bogotá, Cesu, p. 6-11, 2014.

MORIN, E. ¿Hacia un Nuevo contrato social? En: HERNÁNDEZ, Francesc; BELTRÁN, José; MARRERO, Adriana. Teorías sobre sociedad y educación. Valencia: Tirant lo Blanch, p. 966967, 2005.

SANTOS, B.de S. Towards a new legal common sense. London: Butterworth, 2002.

TIBBLE, C. Despojados de Humanidad. En: Arcadia, Publicaciones Semana, Bogotá: n. 124, p. 14-15, 2016.

UNESCO, Declaración sobre la Diversidad Cultural, 2001. <Disponible en: http://portal.unesco.org/es/ev.phpURL_ID $=13179 \& U R L \_D O=D O \_T O P I C \& U R L \_$ SECTION=201.html>. Acesso en: $20 \mathrm{dez} / 2016$.

\section{Sobre o autor}

Andrés Argüello Parra é Professor e investigador da Universidade Santo Tomás da Colômbia. Pósdoutorado em Educação, Ciências Sociais e Interculturalidade da Universidade Santo Tomás (Colômbia). Visiting Scholar do Center for Global Studies and the Humanities in Duke University. Doutor em 
Pedagogia pela Universidade Nacional Autónoma do México (UNAM), com estadia de investigação no Instituto de Sociologia da Universidade de Łodź, na Polônia.

Recebido em fevereiro de 2017.

Aprovado em março de 2017. 